\title{
Phonons in disordered molecular solids: Raman spectra of heavily doped mixed crystals of benzene and perdeuterobenzene*
}

\author{
Hwei-Kwan Hong† and Raoul Kopelman \\ Department of Chemistry, The University of Michigan, Ann Arbor, Michigan 48104
}

(Received 24 August 1972)

\begin{abstract}
Raman spectra of mixed crystals of benzene- $h_{6}$ and benzene- $d_{6}$ have been studied throughout the whole concentration range $(0 \%-100 \%)$. They are discussed in the virtual crystal limit, suitable for small trap depths. It is shown that within this limit: (1) All the phonons are delocalized in mixed crystals; (2) $\mathbf{k}$-designation is still valid, and (3) no appreciable broadening is expected in mixed crystals. In particular, because the trap depths are the same for all three rotations in benzene mixed crystals, it is further inferred that: (1) There is a one-to-one correspondence between the mixed crystal absorption and the pure crystal absortion; (2) spectral line positions depend on the concentration linearly; and (3) the normal coordinates in the mass(or moment of inertia)-weighted coordinates remain unchanged from benzene- $h_{6}$ to mixed crystals to benzene- $d_{6}$. All these conclusions are supported by the experimental data. More general cases, where trap depths are different for different degrees of freedom, are also discussed. Implications of the phonon delocalization in isotopic mixed crystals to the interpretation of phonon sidebands of electronic spectra of isotopic mixed crystals are also pointed out.
\end{abstract}

\section{INTRODUCTION}

Phonons in disordered solids have been studied extensively by physicists since the classic work of Lifshitz $z^{1}$ on the vibrations of imperfect crystals. Most systems studied, however, are atomic crystals where only translational degrees of freedom need to be considered. Molecular crystals constitute a special category of solids in which molecules, rather than atoms, are the building blocks. In general, because of the much stronger internal binding energies of molecules as compared with the weaker intermolecular forces, a rigid body approximation can be justified. Under this assumption, additional rotational degrees of freedom must be taken into account. The theory of disordered molecular solids is, thus, more involved. Further complications come from the anisotropy of intermolecular interactions which are strongly dependent on molecular packing.

Recently, there has been much interest in the study of heavily doped mixed crystals. Investigations in this field range from phonons ${ }^{2}$ to electrons ${ }^{3}$ to excitons. ${ }^{4}$ Partly, the stimulation came from the discovery of switching phenomena in amorphous semiconductors by Ovshinsky. ${ }^{5}$ The discovery, with its potential technological applications and profound theoretical interest, has prompted general studies of disordered solids. Most theories, however, are inapplicable in their present forms to amorphous semiconductors. It is felt that simpler disordered systems, which are amenable to theoretical analysis, would better aid our understanding of general disordered systems. Most theories on alloys ${ }^{6}$ involve the assumption that perturbations are localized and that a well defined "trap depth" can be employed. Furthermore, most theories assume that binary systems are substitutional. Since amorphous semiconductors involve positional disorder, ${ }^{7}$ as well as compositional disorder, ${ }^{7}$ such theories would be inadequate. On the other hand, heavily doped isotopic mixed crystals satisfy very closely these requirements and thus provide convenient systems in which every such theoretical assumption can be safely made.

Mixed crystal studies are also important in their own right, in addition to their general desirability as test case studies of general disordered solids. Mixed crystal studies provide vital information concerning the properties of pure crystals. Various investigations on isotopic mixed crystals have been successful in the past in the elucidation of pairwise interactions, ${ }^{8}$ density-of-states functions, ${ }^{9}$ and dispersion relations ${ }^{10}$ in neat molecular solids such as benzene and naphthalene. Most studies, however, have been centered on excitons rather than phonons.

Literature on either experimental or theoretical studies of lattice phonons in disordered molecular solids is meager. For ordered systems, i.e., neat crystals, the theory of lattice dynamics, and neutron diffraction experiments on molecular solids have just begun to emerge. Pawley and co-workers have done extensive calculations on phonons in hexamethylene-tetramine, ${ }^{11}$ naphthalene, and anthracene. ${ }^{12}$ Dolling and Powell recently published their coherent neutron scattering studies on hexamethylene-tetramine..$^{13}$ More recently, Reynolds, Kjems, and White ${ }^{14 a}$ studied the inelastic neutron scattering of some molecular solids following some earlier studies by Rush and others. ${ }^{14 \mathrm{~b}}$ There are also some neutron scattering works on $\mathrm{Ge}-\mathrm{Si}$ mixed solids, ${ }^{15}$ which are atomic and cubic mixed solids. The corresponding theoretical analysis has been developed by Taylor. ${ }^{16}$

Several theoretical techniques have been developed in dealing with phonons, electrons, and excitons in disordered solids, especially those with substitutional disorder. ${ }^{17}$ These include the moment trace method, ${ }^{18}$ the phase theory, ${ }^{19}$ the negative factor counting method, ${ }^{20}$ and the Green's function method..$^{21}$ Among them, the Green's function method is especially useful in dealing with real 3-dimensional systems. The exact expansion of the mixed crystal Green's function in 
terms of the pure crystal Green's function was obtained by Yonezawa and Matsubara. ${ }^{22}$ An approximation called the Coherent Potential Approximation (CPA) was independently proposed by Soven, ${ }^{23}$ Taylor, ${ }^{16}$ and Onodera and Toyozawa. ${ }^{24}$ Velicky et $a l .{ }^{25}$ later demonstrated that such an approximation really amounts to a self-consistent single-site approximation. The application of CPA to Frenkel exciton states of mixed molecular solids with nontrivial interchange symmetry ${ }^{26}$ has been carried out by Hong and Robinson, ${ }^{27}$ Dubovskii and Konobeev ${ }^{28}$ and by Hoshen and Jortner. ${ }^{29}$ In most cases, however, an isolated band was involved.

Recently, we have extended the general principle of self-consistent single-site approximation to the case of interacting bands. ${ }^{30}$ The theory was developed for electronic excitons with bands that are coupled either via vibronic or spin-orbit coupling mechanisms, for vibrational excitons with either Fermi resonance ${ }^{31}$ or degeneracy in the gas phase, for triplet excitons with interacting magnetic sublevels, and for phonons in molecular solids in general. Parallel to these theoretical developments, we present here experimental data on the Raman spectra of lattice phonons in heavily doped mixed crystals of benzene and perdeuterobenzene.

The mixed crystal Raman spectra were studied throughout the whole concentration range $(0 \%-100 \%)$. They are analyzed in the light of our new theoretical development. In particular, the weak perturbation was treated by assuming an energy-independent self-energy and thus avoiding the difficult energy-dependent term. This is the so called virtual crystal limit, ${ }^{25}$ suitable for weak perturbations. It is found that the mixed crystal phonons can be classified as the amalgamated case, borrowing the terminology from discussions of electrons and excitons in disordered solids. ${ }^{24}$ It is also concluded that: (1) phonons are delocalized in isotopic mixed crystals, in contrast to the localized phonons observed in some chemically mixed crystals ${ }^{32} ;(2) \mathbf{k}$ designations are still valid, indicating a relatively small scrambling of the $\mathbf{k}$ states; (3) Within this virtual crystal limit, there will be no borrowing of intensities from the far ir active lines because, for the $\mathbf{k}=0$ states, $g$ modes (Raman active), and $u$ modes (far ir active) are decoupled; (4) Perturbations introduced by the presence of impurities are rather mild and the normal coordinates in the mass (or moment of inertia)-weighted coordinates remain relatively unchanged in going from one given neat component to admixtures and from them to another component. These conclusions will have important implications towards the discussion of phonon sidebands in electronic spectra of mixed crystals, towards excitation transfer, and radiationless transitions and towards normal coordinate analyses of ordered (neat) systems.

\section{EXPERIMENTAL}

Reagent grade benzene- $h_{6}$ was obtained from Baker and $99.5 \%$ (atom $\%$ ) benzene- $d_{6}$ was obtained from

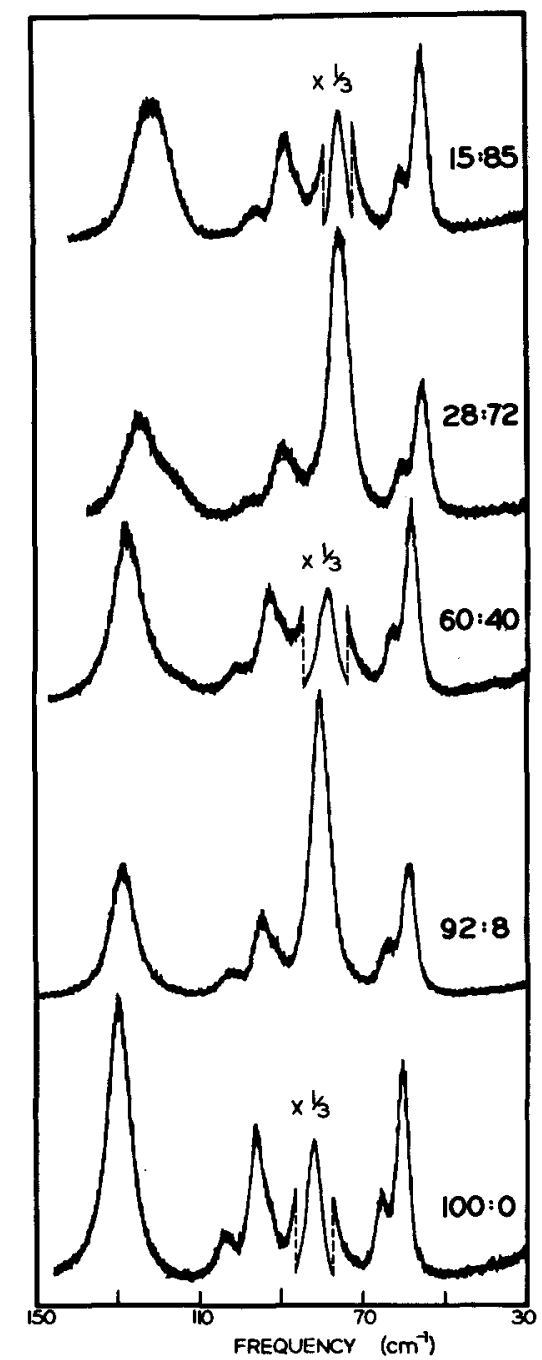

FIG. 1. Raman spectra of mixed crystals of benzene- $h_{6}$ and benzene- $d_{6}$ at $104^{\circ} \mathrm{K}$. The concentrations are given as benzene- $h_{6}$ : benzene- $d_{6}$. Notice the scale change for the most intense Raman line in some of the spectra.

Merck, Sharp, and Dohme and used without further purification. Mixed crystals were grown from the melt in a capillary. The samples were placed in a silvered double-jacket tubing and cooled by a flow of nitrogen vapor evaporated from a liquid nitrogen reservoir by passing current through a resistor. The temperature of the samples in the system was measured to be about $104^{\circ} \mathrm{K}$ with a copper-constantan thermocouple. Spectra were taken photoelectrically on a Ramalog-Spex double spectrometer (model 1401). An argon ion laser from Coherent Radiation Laboratory was used as an excitation source. The laser emission at $4880 \AA$ (with an appropriate interference filter) was used as the excitation line. The spectral resolution was about $1 \mathrm{~cm}^{-1}$. The concentrations of the mixed crystals were determined by comparing the intensities of Raman lines of benzene- $h_{6}$ and benzene- $d_{6}$ at 990 and $944 \mathrm{~cm}^{-1}$, respectively. The spectra are shown in Fig. 1. 


\section{RESULTS AND DISCUSSIONS}

\section{A. The Interacting-Band Picture}

To simplify our discussions, we shall ignore the couplings between the internal modes (vibrational excitons) and the external modes (lattice phonons). This is the rigid-body approximation. Recently, Pawley and Cyvin ${ }^{12}$ considered the effect of nonrigidity on the normal modes of crystalline naphthalene. For naphthalene, the highest external mode and the lowest internal mode are quite close, 146 and $176 \mathrm{~cm}^{-1}$, respectively, so such an effect would be more important. On the other hand, for crystalline benzene, $\nu_{16}$, which is the lowest internal mode and lies at ${ }^{33} 352 \mathrm{~cm}^{-1}$ in $\mathrm{C}_{6} \mathrm{D}_{6}$, is well separated from the highest external mode of about $130 \mathrm{~cm}^{-1}$, in $\mathrm{C}_{6} \mathrm{H}_{6}$. Thus it seems that the separation of vibrational excitons from phonons can be justified. Therefore, we shall only deal with the 24 external modes out of a total of 144 normal modes (for the Brillouin zone center). Further separations cannot be achieved. It is true that, for the pure crystal Brillouin zone center $(\mathbf{k}=\mathbf{0})$ states, there is no coupling between the rotations and translations. However, Cochran and Pawley, ${ }^{34}$ in their treatment of hexamethylene-tetramine, pointed out that, for general $\mathbf{k}$ states, such couplings must be considered. It is well known that the introduction of impurities causes the mixing of $\mathbf{k}$-states of the pure crystal. Since rotations and translations are coupled in the pure crystal, there is no a priori reason why we should consider only the rotational modes, although we are discussing only the Raman spectra of mixed crystals. We do show later that such couplings are indeed zero in the virtual crystal limit suitable for mixed crystals of benzene. Nevertheless, a general discussion calls for the full consideration of both types of motion.

To circumvent the difficulty of considering several interacting bands, Prasad and Kopelman ${ }^{35}$ proposed the concept of librational exciton for phonons in crystalline naphthalene. Such a concept, although useful for crystalline naphthalene, is inadequate for the present system (vide infra). Basically, if the rotations and translations can be separated and if the three rotations are only coupled weakly, each degree of freedom can be treated independently. In such cases, the exciton picture would be appropriate. The mixed crystal theory for excitons, presented by Hong and Robinson, ${ }^{27}$ Dubovskii and Konobeev ${ }^{28}$ and by Hoshen and Jortner, ${ }^{29}$ can then be applied to phonons. Basic concepts in the exciton formulation, such as pairwise interactions, Davydov splittings etc., can then be transferred to the phonon case without any difficulties. In the present case, the lattice dynamics has been treated by Harada and Shimanouchi, ${ }^{36}$ Ito and Shigeoka, ${ }^{37}$ Oliver and Walmsley, ${ }^{38}$ Bernstein ${ }^{39}$ and very recently by Taddei et $a l . .^{40}$ Even for the Raman-active $(\mathrm{k}=0)$ phonons there is a considerable amount of mixing among the three basic rotations $\left(R_{x}, R_{y}, R_{z}\right)$. In Fig. 2 we classified the optically active normal modes as being essentially $R_{x}, R_{y}$, or $R_{z}$ if they have a large coefficient (projection) of either $R_{x}, R_{y}$ or $R_{z}$. It can be easily seen that the bands are entwined. In other words, we can no longer consider a band as being formed by a single basis function $\left(R_{x}, R_{y}\right.$, or $R_{z}$ in the phonon case and free molecular functions or site functions in the exciton case $\left.^{41}\right)$. We are forced to consider the interacting bands in crystalline benzene. In the corresponding mixed crystal theory, all six bands ${ }^{42}$ must be considered simultaneously.

\section{B. The Coherent Potential Approximation for Interacting Bands in the Weak Perturbation Limit}

The general theory of phonons in molecular solids with nontrivial interchange symmetry has been presented by Hong and Kopelman ${ }^{30}$ within the selfconsistent single-site single band approximation. This is the CPA theory for interacting bands. Two approaches were proposed, one more rigorous and the other more approximate. In the first approach, the mixed crystal Green's function $\left(R_{n \alpha f}\right)$ and the self-energy $\left(\Sigma_{f}\right)$ for a particular degree of freedom $f$ were given by

$$
\begin{gathered}
R(\mathbf{k})_{\alpha f, \alpha^{\prime} f^{\prime}}=G^{0}(\mathbf{k})_{\alpha f, \alpha^{\prime} f^{\prime}} \\
+\sum_{\alpha \prime \alpha^{\prime \prime}} \sum_{f^{\prime \prime}} G^{0}(\mathbf{k})_{\alpha f, \alpha^{\prime \prime} f^{\prime \prime}} \Sigma_{f^{\prime \prime}} R(\mathbf{k})_{\alpha^{\prime \prime} f^{\prime \prime}, \alpha^{\prime} f^{\prime},} \\
R_{n \alpha f}=N^{-1} \sum_{k} R(\mathbf{k})_{\alpha f, \alpha f}, \\
\Sigma_{f}=\Sigma_{f}^{\prime}+C_{B} \eta_{A}{ }^{f} \Delta^{f} \omega^{2}, \\
\Sigma_{f}^{\prime}=C_{A} C_{B}\left(\eta_{A}^{f} \Delta^{f} \omega^{2}\right)^{2} /\left[R_{n \alpha f^{-1}}+\left(C_{B}-C_{A}\right)\left(\eta_{A}^{f} \Delta^{f} \omega^{2}\right)+\Sigma_{f}^{\prime}\right],
\end{gathered}
$$

and

$$
\begin{aligned}
& G^{0}(\mathbf{k})_{\alpha f, \alpha^{\prime} f^{\prime}}=\left(\eta_{A} f_{A^{\prime}} f^{\prime \prime}\right)^{-1 / 2} \\
& \quad \times \sum_{j}\left[B_{\alpha f}(\mathbf{k}, j) B_{\alpha^{\prime} f^{\prime}} *(\mathbf{k}, j) / \omega^{2}-\omega^{2}(\mathbf{k}, j)\right],
\end{aligned}
$$

where $\alpha$ is the sublattice index: For example, the benzene crystal has four molecules per primitive unit cell and therefore, $\alpha=\mathrm{I}$, II, III, IV. $f$ denotes the degree of freedom: In the benzene crystal, $f$ would be $T_{x}, T_{y}$, $T_{z}, R_{x}, R_{y}$, and $R_{z}$, where $T$ is translation and $R$ is rotation. $\eta_{A} f$ is either the mass (where $f=T$ ) or the moment of inertia (where $f=R$ ) for the pure crystal of component $A . C_{A}, C_{B}$ are, respectively, the molar concentrations of $A$ and $B$ in the mixed crystal. $\Delta_{f}$ measures the perturbation strength and was defined as $\left(\eta_{A}^{f}-\eta_{B}^{f}\right) / \eta_{A}{ }^{f} . G^{0}(\mathbf{k})_{\alpha f, \alpha^{\prime} f^{\prime}}$ is the pure crystal Green's function of component $A$, which is completely determined if the pure crystal normal frequency $\omega(\mathbf{k}, j)$ and the normal modes $|\mathbf{k}, j\rangle$ are known. $|\mathbf{k}, j\rangle$ is, of course, defined as ${ }^{43}$

$$
\begin{aligned}
& |\mathbf{k}, j\rangle=(N)^{-1 / 2} \sum_{n} \sum_{\alpha} \sum_{f} B_{\alpha f}(\mathbf{k}, j) \\
& \times \exp \left(i \mathbf{k} \cdot \mathbf{R}_{n \alpha}\right)\left(\eta_{A^{f}}\right)^{1 / 2} u_{\alpha f}(\mathbf{n}) \\
& =\sum_{\boldsymbol{\alpha}} \sum_{f} B_{\alpha f}(\mathbf{k}, j)\left(\eta_{A^{f}}\right)^{\mathbf{1} / \mathbf{2}} u(\mathbf{k}, \boldsymbol{\alpha}, f) \text {, }
\end{aligned}
$$


where $j$ is the branch index and $u_{\alpha f}(\mathbf{n})$ is the displacement (either angular or linear) of the molecule at site $\mathbf{n}, \boldsymbol{\alpha}$ in the $f$ th degree of freedom. The definition of $u(\mathbf{k}, \alpha, f)$ is obvious ${ }^{30}$ from Eqs. (6). Since $G^{0}(\mathbf{k})_{\alpha f, \alpha^{\prime} f^{\prime}}$ is assumed to be known, Eqs. (1)-(5) really define two simultaneous equations for the two unknowns, $R_{n \alpha f}$ and $\Sigma_{f} . \Sigma_{f}^{\prime}$ is defined only for convenience. ${ }^{30}$

Similarly, in the second approach, the Green's function and the self-energy are given as

$R_{n \alpha f}=\left(N \eta_{A}\right)^{-1} \sum_{k} \sum_{j}\left[\left|B_{\alpha f}(\mathbf{k}, j)\right|^{2} / \omega^{2}-\omega^{2}(\mathbf{k}, j)-\Sigma\right]$

and

$$
\begin{aligned}
& \sum_{f}\left(\eta_{A}^{f}+\eta_{A}^{\prime} R_{n \alpha f} \Sigma_{f}^{\prime}\right)^{-1} \\
& \quad \times\left(\Sigma_{f}^{\prime}-\frac{C_{A} C_{B}\left(\eta_{A}^{f} \Delta^{f} \omega^{2}\right)^{2}}{R_{n \alpha f^{-1}}+\left(C_{B}-C_{A}\right)\left(\eta_{A}^{f} \Delta^{f} \omega^{2}\right)+\Sigma_{f}^{\prime}}\right)=0 .
\end{aligned}
$$

In this approach, however, the self-energy is assumed to be

$$
\eta_{A}^{f} \Sigma=\Sigma_{f}^{\prime}+C_{B} \eta_{A}^{f} \Delta^{f} \omega^{2} .
$$

Careful consideration would show that Eqs. (7)-(9) define only one unknown, namely, $\Sigma . \Sigma_{f}^{\prime}$ can, then, be obtained from Eq. (9) and $R_{n \alpha f}$ determined from Eq. (7). Furthermore, to solve Eqs. (7) and (8), the only input to the theory is the projection on $f$ of the densityof-states $\rho_{f}$ defined as

$$
\rho_{f}(\omega)=N^{-1} \sum_{k} \sum_{j}\left|B_{\alpha f}(\mathbf{k}, j)\right|^{2} \delta[\omega-\omega(\mathbf{k}, j)] .
$$

Equation (7) can, then, be recast as

$$
R_{n \alpha f}=\left(\eta_{A} f\right)^{-1} \int\left[\rho_{f}\left(\omega^{\prime}\right) d \omega^{\prime} /\left(\omega^{2}-\omega^{\prime 2}-\Sigma\right)\right] .
$$

In our previous paper ${ }^{30}$ we have shown that in the limit of infinite dilution our formulation gives only approximate single-impurity levels. This is an inherent feature of such a single-site single band approach..$^{30} \mathrm{We}$ also consider here the first approach as more rigorous and the second as more approximate. We elaborate on this point here because it is relevant to our discussions later. The reason why our first approach is more rigorous is because it gives the correct eigenstates of the pure crystal of component $B$, as we approach the limit $C_{B} \rightarrow 1$. As we explained in our previous paper, ${ }^{30}$ the CPA theory for one band only can be constructed by treating components $A$ and $B$, on an equal footing. For interacting bands, one has to start from either the pure crystal eigenstates of $A$ or those of $B$ depending on which one of the two sets is known (for convenience, we have assumed in this and the other paper ${ }^{30}$ that we know the pure crystal eigenstates of component $A$ ). It should be obvious that the eigenstates of $B$ are considered as unknowns to be predicted from the theory rather than the input parameters. A more rigorous formulation should, then, be the one which has the right behavior near $C_{B} \rightarrow 1$. In our previous paper, ${ }^{30}$ we

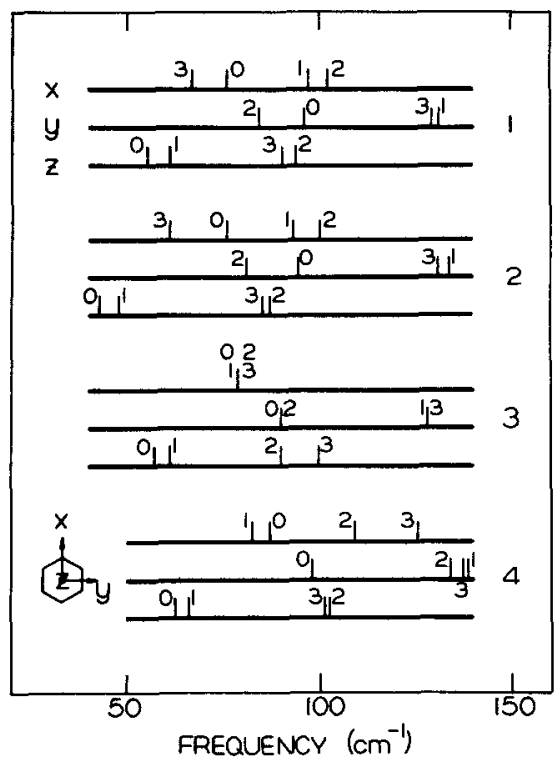

FIG. 2. The interacting-band picture for benzene lattice phonons as demonstrated by various theoretical calculations of: (1) Taddei t al. (Ref. 40), (2) Oliver and Walmsley (Ref. 38), (3) Ito and Shigeoka (Ref. 37) and (4) Bernstein (Ref. 39). $x, y$, and $z$ refer to the three basic rotations around the three principal molecular axes shown in the lower left corner. We use the convention suggested by Oliver and Walmsley and by Ito and Shigeoka. Taddei $e t a l$. and Bernstein used different conventions. Their results have been converted to the present convention in preparing (1) and (4). The number associated with each line denotes the symmetry species: $0=a_{1 g} ; 1=b_{1 g} ; 2=b_{20} ; 3=b_{8 g}$, respectively.

have defined

$$
Q_{n \alpha f}=V_{n \alpha f} /\left(1-R_{n \alpha f} V_{n \alpha f}\right),
$$

where $V_{n \alpha f}$ is the fluctuation related to the singleconfiguration perturbation matrix $\delta \mathrm{L}$ and the average perturbation matrix $\boldsymbol{\Sigma}$ by the following expression:

$$
\delta \mathbf{L}-\mathbf{\Sigma}=\sum_{n} \sum_{\alpha} \sum_{f} V_{n \alpha f} .
$$

If all the molecules of $A$ are replaced by molecules of $B$, we again have an ordered system with no fluctuations. Therefore, $V_{n \alpha f}=0$ and $Q_{n \alpha f}=0$. Since we have used the self-consistent conditions ${ }^{30}$

$$
\left\langle Q_{n \alpha f}\right\rangle=0,
$$

for the first approach and

$$
\sum_{f}\left\langle Q_{n \alpha f} / \eta_{A}{ }^{f}\right\rangle=0
$$

for the second approach, it is apparent that the selfenergy based on the first approach has the right behavior in such a limit whereas that of the second approach does not have it, necessarily. These features will become clear as we proceed to discuss the mixed crystal phonons of benzene- $h_{6}$ and benzene- $d_{6}$.

In this paper, we do not make full usage of either Eqs. (1) to (5) or Eqs. (7) and (8), but rather examine the behavior of $\Sigma_{f}$ and $R_{n \alpha f}$ in the weak perturbation limit which is suitable for the mixed crystals of benzene 
and perdeuterobenzene. The perturbation strength, as measured by $\Delta_{f}$ for any benzene translation, is $-6 / 78=$ -0.077 . Using the known benzene molecular structure, ${ }^{44}$ $\Delta_{f}$ for each rotation is calculated to be $-36.0 / 175.1=$ -0.21 . It is, therefore, certainly appropriate to consider the mixed crystals in the weak perturbation limit. It is easily seen from Eqs. (3) and (4) that, when $\Delta \rightarrow 0, \Sigma_{f}^{\prime} \rightarrow 0$, and therefore,

$$
\Sigma_{f} \rightarrow C_{B} \eta_{A}{ }^{f} \Delta^{f} \omega^{2} .
$$

Similarly, From Eqs. (8) and (9), as $\Delta_{r} \rightarrow 0$, we have

$$
\sum_{f}\left(\Sigma_{f}^{\prime} / \eta_{A} f\right) \rightarrow 0
$$

or

$$
\Sigma \rightarrow(1 / F) \sum_{f} C_{B} \Delta^{f} \omega^{2}
$$

where $F$ is the total number of degrees of freedom. The above is, indeed, the virtual crystal limit, ${ }^{25}$ similar in spirit to the first order perturbation theory, where we use the zero order wavefunctions (the pure crystal normal coordinates) to obtain the first order correction to the energy (normal frequency). The fluctuating potential introduced by the presence of the impurity is, of course, completely ignored and $\mathbf{k}$ designation of states is still valid in such a limit. Within this limit, the broadenings of the spectral lines are not accounted for. Experimental data show very little broadening (vide infra), consistent with this simplified model.

We are now in a position to calculate the mixed crystal Green's function without any knowledge of either the density-of-states function or the dispersions relations of the pure crystal. To obtain the spectrum, we have to evaluate the mixed crystal Green's function in the $|\mathbf{k}, j\rangle$ representation. This can be accomplished by transforming Eq. (1) to the $|\mathbf{k}, j\rangle$ representation, using Eq. (6b). Since $G^{0}$ is diagonal in such a representation, the mixed crystal Green's function takes up a simpler form

$$
R_{k j, k j^{\prime}}=G^{0}(\mathbf{k}, j) \delta_{j j^{\prime}}+\sum_{j^{\prime \prime}} G^{0}(\mathbf{k}, j) \Sigma_{k j, k j^{\prime \prime}} R_{k j^{\prime \prime}, k j^{\prime}},
$$

where

$$
\Sigma_{k j, k j^{\prime \prime}}=\sum_{\alpha} \sum_{f} B_{\alpha f^{*}}(\mathbf{k}, j) B_{\alpha f}\left(\mathbf{k}, j^{\prime \prime}\right) C_{B} \Delta^{f} \omega^{2}
$$

and

$$
G^{0}(\mathbf{k}, j)=\left[\omega^{2}-\omega^{2}(\mathbf{k}, j)\right]^{-1} .
$$

$\boldsymbol{R}_{k j, k j^{\prime}}$ is, in general, not diagonal! However, if one is only interested in the $\mathbf{k}=\mathbf{0}$ states (i.e., the spectrum), some simplification can be made. $\Sigma_{0 j, 0 j}$, is simply

$$
\Sigma_{0 j, 0 j^{\prime}}=\sum_{\alpha} \sum_{f} B_{\alpha f^{*}}(\mathbf{0}, j) B_{\alpha f}\left(\mathbf{0}, j^{\prime}\right) C_{B} \Delta^{f} \omega^{2} .
$$

At this point, some important observations can be made.

(1) There will be no borrowing of intensities from farinfrared active modes by the Raman active modes and vice versa. To see this, we use $l$ and $m$ as respective branch indices for $g$ modes and $u$ modes. Furthermore, $f=R$, for rotation, and $f=T$, for translation. Therefore,

$$
\begin{aligned}
\Sigma_{0 l, 0 m}=\sum_{\alpha}\left(\sum_{R} B_{\alpha R}{ }^{*}(0, l) B_{\alpha R}(\mathbf{0}, m) C_{B} \Delta^{R} \omega^{2}\right. \\
\left.+\sum_{T} B_{\alpha T^{*}}(\mathbf{0}, l) B_{\alpha T}(\mathbf{0}, m) C_{B} \Delta^{T} \omega^{2}\right) .
\end{aligned}
$$

The first term on the rhs is zero because ${ }^{36} B_{\alpha R}(0, m)=0$. Similarly, the second term is zero because ${ }^{36} B_{\alpha T}(\mathbf{0}, l)=0$. Consequently, $\Sigma_{0 l, 0 m}=0$ and no coupling exists between the far-infrared and Raman spectra in the mixed crystal. This gives a sum rule: Within the virtual crystal limit, the total intensity of the Raman spectra of the mixed crystal is equal to that of the pure crystal. The same rule is true for the far-infrared intensities. (2) In the case of naphthalene, or similar systems where bands are isolated, we can associate $j$ with $f$. Therefore, $B_{\alpha f}(0, j)=1 / \sigma$ and $B_{\alpha f}{ }^{\prime}(0, j)=0$, where $\sigma$ is the total number of molecules per unit cell, if $j$ belongs to $f$ but not to $f^{\prime}$. As a consequence,

$$
\Sigma_{0 j, 0 j^{\prime}}=0,
$$

if $j$ and $j^{\prime}$ belong to different degrees of freedom; however, one also gets

$$
\Sigma_{0 j, 0 j^{\prime}}=0,
$$

if $j$ and $j^{\prime}$ belong to the same degree of freedom. The last result comes from the fact that $\Delta^{f}$ is the same for both $j$ and $j^{\prime}$ and

$$
\sum_{\alpha} B_{\alpha f}{ }^{*}(0, j) B_{\alpha f}\left(0, j^{\prime}\right)=0 .
$$

It is, thus, concluded that

and

$$
\begin{gathered}
\Sigma_{0 j, 0 j^{\prime}}=C_{B} \Delta^{f} \omega^{2} \delta_{j j^{\prime}}, \\
R_{0 j, 0 j^{\prime}}=\delta_{j j^{\prime}} /\left[\omega^{2}-\omega^{2}(0, j)-C_{B} \Delta^{f} \omega^{2}\right] .
\end{gathered}
$$

The spectral lines are determined by locating the poles of the Green's function, i.e.,

$$
\omega=\omega(0, j) /\left(1-C_{B} \Delta^{f}\right)^{1 / 2} .
$$

This gives a line which is shifted but has the same intensity as the one in the pure crystal. The conservation of intensity can be applied to each individual line. (3) This is the case of crystalline benzene where $\Delta$ is the same for all three rotations (it is always the same for all the translations). We can denote $\Delta^{f}=\Delta^{R}$, if $f$ is a rotation, and $\Delta^{f}=\Delta^{T}$, if $f$ is a translation.

It is obvious that we obtain the same results as in case (2) for very different reasons, namely the fact that $\Delta^{f}=\Delta^{R}$ (i.e., the special case of a symmetric planar top, which in this respect gives the same answer as a spherical top). It should be emphasized that if $\Delta$ is different for different rotations $\Sigma_{0 j, 0 j^{\prime}}$ will not vanish. This would lead to a scrambling of intensities among different Raman lines. However, the far-infrared spectrum of mixed crystals should still have the same intensity pattern as that of the pure crystal because $\Delta$ is always 
equal for all three translations. In conclusion, for Raman active modes, we have

$$
\omega=\omega(0, l) /\left(1-C_{B} \Delta^{R}\right)^{1 / 2},
$$

where $l$ runs from one to twelve for all the $g$ modes in crystalline benzene- $h_{6}$, and

$$
\omega=\omega(0, m) /\left(1-C_{B} \Delta^{T}\right)^{1 / 2},
$$

for the translational modes. It is clear that, when $C_{B} \rightarrow 1$,

$$
\omega=\omega(0, l) /\left(1-\Delta^{R}\right)^{1 / 2}=\left(\eta_{A}^{f} / \eta_{B}^{f}\right)^{1 / 2} \omega(0, l),
$$

which are exactly the pure crystal frequencies of benzene- $d_{6}$ as one would expect from the pure crystal theory. ${ }^{37}$ We have a mixed crystal theory which is exact at both concentration limits $\left(\mathrm{C}_{B}=1\right.$ and $C_{A}=1$ ) (for example, see Fig. 3).

The situation is somewhat different when the second approach is used. The self-energy associated with a subband $f$ is given as $\eta_{A} f \Sigma$, where $\Sigma$ is nothing but the average trap depth. In the virtual crystal limit, $\Sigma$ can be written from $\mathrm{Eq}$. (15) as

$$
\Sigma=\frac{1}{2}\left(\Delta^{R}+\Delta^{T}\right) C_{B} \omega^{2} .
$$

$\Sigma_{k j, k j^{\prime}}$, now, becomes

$\Sigma_{k j, k j^{\prime}}=C_{B} \Sigma \omega^{2} \sum_{\alpha} \sum_{f} B_{\alpha f^{*}}{ }^{*}(\mathbf{k}, j) B_{\alpha f}\left(\mathbf{k}, j^{\prime}\right)=C_{B} \Sigma \omega^{2} \delta_{j j^{\prime}}$

It is diagonal in the $|\mathbf{k}, j\rangle$ representation! We have, therefore,

$$
G_{k j, k j^{\prime}}=\delta_{j j^{\prime}} /\left[\omega^{2}-\omega^{2}(\mathbf{k}, j)-C_{B} \Sigma \omega^{2}\right] .
$$

The mixed crystal levels are given by

$$
\omega=\omega(\mathbf{k}, j) /\left(1-C_{B} \Sigma\right)^{1 / 2} \text {. }
$$

Contrary to results of the first approach, we have a situation where not only $\mathbf{k}$ but also $j$ is a good quantum number for the mixed crystals. There is a one-to-one correspondence between the mixed crystal levels and the pure crystal levels as given by Eq. (27). For $\mathbf{k}=\mathbf{0}$ states, we have

$$
\omega=\omega(0, j) /\left(1-C_{B} \Sigma\right)^{1 / 2},
$$

which, of course, does not predict the right benzene- $d_{6}$ frequencies as $C_{B} \rightarrow 1$ (for example, see Fig. 5).

Notice that although the second approach appears to be inferior in this particular example, when it comes to the full usage of Eqs. (1)-(5), or Eqs. (7)-(9), the second approach does contain some attractive features because only the partial density-of-states functions are called for.

\section{Experimental Data and Their Interpretation}

Mixed crystal Raman spectra of benzene and perdeuterobenzene at $104^{\circ} \mathrm{K}$ are shown in Fig. 1. Our spectra are of better quality than those of Ito and Shigeoka $^{37}$ which were taken with $\mathrm{Hg}$ excitation. In

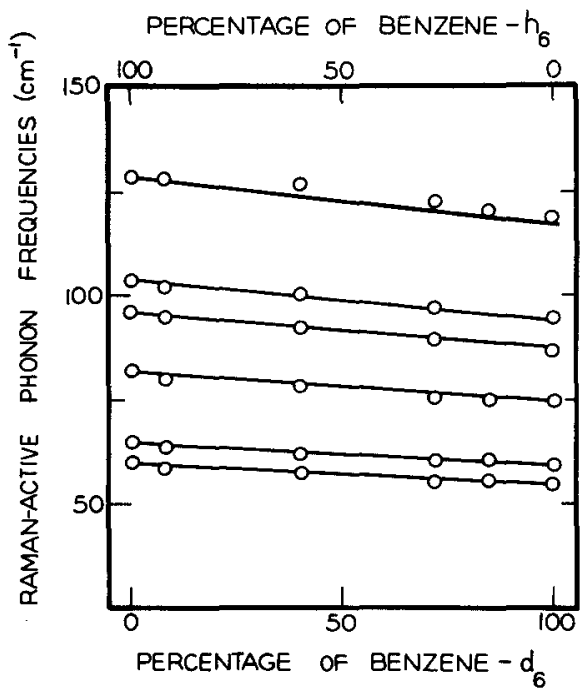

FIg. 3. Comparison of spectral line positions with the theoretical predictions based on the virtual crystal model. Open circles are experimental data and solid lines are theoretical results. The solid lines are prepared by using the known frequencies of benzene- $h_{6}$ and $\mathrm{Eq}$. (21) [or, equivalently, Eq. (29) for small $\Delta^{R}$ ]. $\Delta^{R}=-0.21$. Notice that we did not draw the lines by joining the benzene- $h_{6}$ frequencies with the benzene- $d_{6}$ frequencies (see text). However, the more rigorous approach was used, which "guarantees" that lines would end at the right benzene- $d_{6}$ frequencies (see text).

particular, we have been able to resolve the lowest lines as 60 and $65 \mathrm{~cm}^{-1}$, which Ito and Shigeoka could not resolve even at $77^{\circ} \mathrm{K}$. Because we used polycrystalline samples with polarization determinations, we did not resolve the line at $96 \mathrm{~cm}^{-1}$ which was reported recently by Bonadeo et al..$^{45}$ to consist of two lines, 90 and 92 $\mathrm{cm}^{-1}$, at $140^{\circ} \mathrm{K}$. The assignments of various lines have been done recently by Bonadeo et $a .^{45}$ and will not be repeated here.

The first important observation (see Fig. 1) is the lack of any appreciable absorption below $60 \mathrm{~cm}^{-1}$. This is predominantly the translational region. The lack of any stealing of intensity by translations from rotations is strong evidence that the virtual crystal approach used here is well justified. Furthermore, the spectral lines are sharp. The 28:72 sample shows somewhat broader lines, especially for the highest frequency component. This might be due to poor crystal quality rather than something fundamental. As we have shown in the last section [Eq. (21)], for the first approach,

$$
\omega=\omega(0, l) /\left(1-C_{B} \Delta^{R}\right)^{1 / 2} .
$$

Since $|\Delta|=|-0.21|$ is a small quantity (compared to one), we can approximately put

$$
\omega=\omega(0, l)\left(1+\frac{1}{2} C_{B} \Delta^{R}\right) .
$$

Thus, a practically linear dependence of spectral lines on concentration is predicted. In Fig. 3, we calculated the spectral line positions using the known benzene- $h_{6}$ frequencies, $\Delta=-0.21$, and Eq. (21). The agreements 


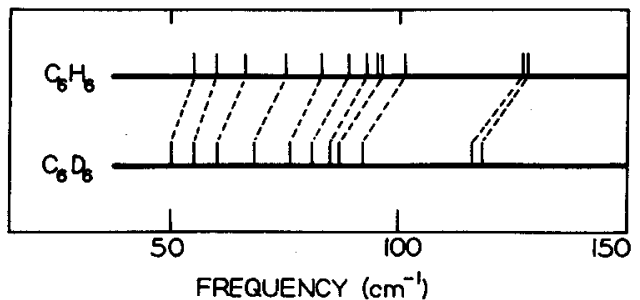

FIG. 4. Raman frequencies of benzene- $h_{6}$ and benzene- $d_{6}$ as calculated by Taddei et al. (Ref. 40). The figure was prepared so as to demonstrate that the normal coordinates in the mass- (or moment of inertia)-weighted coordinates are the same for both compounds. Notice that the dotted lines, which connect benzene- $h_{6}$ frequencies with their corresponding benzene- $d_{6}$ frequencies, never cross each other. Each benzene- $d_{6}$ frequency is related to each benzene- $h_{6}$ frequency by a constant ratio [see Eq. (23)].

are, indeed, excellent. Notice that although $R_{x}, R_{y}$, and $R_{z}$ are mixed, we have a pseudosingle band because $\Delta^{R}$ is independent of the degrees of freedom. This is why the benzene- $d_{6}$ frequencies are related to those of benzene- $h_{6}$ by a simple constant ratio.

The good agreements between the theory and the experiments indicate that our assumption of $\Sigma_{f}^{\prime}=0$ is well justified. It also proves that our mixed crystals are simple substitutionally disordered systems, i.e., the force constants of the mixed crystals are the same as those of the pure crystals. It follows immediately that: (1) $\mathbf{k}$-designation is still valid in the mixed crystal; (2) all the phonons are delocalized; (3) the normal coordinates in the mass(or moment of inertia)-weighted coordinates remain unchanged in going from benzene- $h_{6}$ to mixed crystals to benzene- $d_{6}$. The last aspect is also supported by recent calculations of Taddei et al..$^{40}$ which we reproduce in Fig. 4 . It is obvious from Fig. 4 that there is no crossing of normal coordinates.

We have used also the second approach to calculate the location of Raman lines in mixed crystals. The proper equation for the optically active levels is a slightly modified version of Eq. (29), namely, from Eq. (28),

$$
\omega=\omega(0, l)\left(1+\frac{1}{2} C_{B} \Sigma\right),
$$

where $\Sigma=-0.14$ for benzene. This is to be compared with the exact result, $\Delta^{R}=-0.21$. Results from using the second approach are shown in Fig. 5. Because of the smallness of $\Sigma$ (and $\Delta^{R}$ ), deviations appear to be tolerable. Only few branches show larger deviations, despite the fact that the translational trap depth is only one third of the rotational trap depth.

We have here investigated only the Raman spectra of mixed crystals. We expect that the far-infrared spectra (due to translations) would behave quite similarly. $\Delta^{T}$ for translations amounts to only $\mathbf{- 0 . 0 7 7}$. A virtual crystal approach should also be applicable and a linear dependence of spectral lines on the concentration can be predicted. It would be interesting to investigate other systems where $\Delta^{r}$ is a constant but not $\Delta^{R}$. According to the theory presented here, there would be no scrambling of intensities in the far-infrared spectra; however, there should occur a redistribution of intensities among the Raman lines, depending on how $\Delta^{R}$ varies among the different degrees of freedom.

\section{Application to Phonon Sidebands}

Finally, we would like to comment on the implication of delocalized phonons to the phonon sideband in the electronic spectra of mixed crystals. Chereson et al..$^{32}$ recently studied the phonon sidebands in the absorption and emission spectra of naphthalene in durene and in biphenyl. They attributed the phonon structures to in band localized modes of the impurities. The situation is quite different in the case of isotopic mixed crystals. Since phonons are delocalized and, to a large extent, resemble phonons of the pure crystals, the phonon sideband in isotopic mixed crystals would be the phonon density-of-states function modified by the exciton-phonon coupling function. ${ }^{46}$ Qualitatively, one can argue that there is a transfer of pseudomomentum between excitons and phonons during the transition. In most experiments, such as the emission experiment, we generally start with a localized exciton (or exciton with various pseudomomenta $h \mathbf{k}$ ). Such an exciton will decay with the emission of a photon $(\mathbf{k}=0)$ and we end up with phonons with various momenta $h \mathbf{k}$ as the final states. If the probability of such conversions of momentum is independent of $\mathbf{k}$ (exciton-phonon coupling is independent of $\mathbf{k}$ ), then the corresponding transition would also be $\mathbf{k}$-independent. The phonon sideband will therefore reflect the phonon density-of-states function. This effect has been utilized by Kopelman et $a l .{ }^{46}$ to study the phonon density-of-states function in

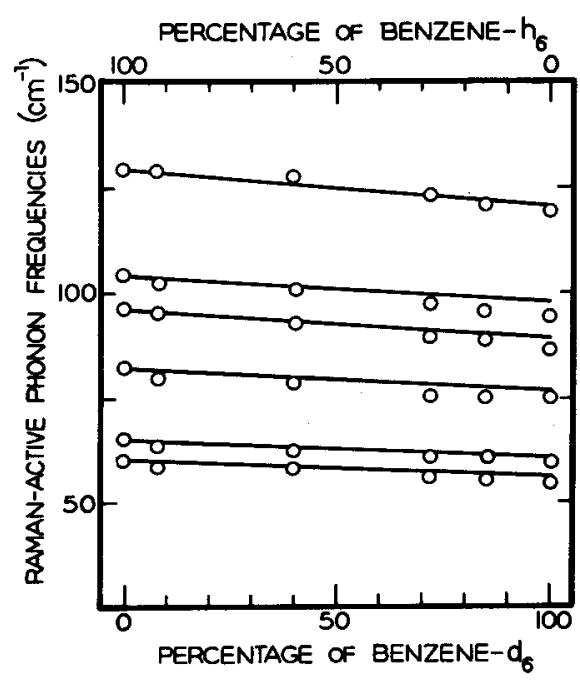

FIG. 5. Comparison between the spectral line positions (open circles) and theoretical predictions based on the more approximate approach (solid lines). An average trap depth, $\Sigma=-0.14$, was used. This particular approach [Eq. (28), or Eq. (30)] does not predict the correct benzene- $d_{b}$ frequencies (see far right), however, discrepancies are small and appear to be tolerable. 
naphthalene. It should thus be also interesting to study the phonon sideband in mixed benzene crystals, having learned that the phonons are delocalized in these systems.

\section{SUMMARY}

In this paper, several conclusions have been drawn: (1) Phonons in mixed crystals of benzene and perdeuterobenzene can be treated in the virtual crystal limit. (2) This predicts a nearly linear concentration dependence of spectral lines, in agreement with the experimental data. (3) Phonons are, thus, delocalized and $\mathbf{k}$ designation is, to a large extent, still valid in this disordered system. (4) Because the perturbation $\Delta^{R}$ is a constant for all rotations, each Raman line in the isotopic mixed crystal has the same intensity as that of the corresponding line in the pure crystal. (5) The normal coordinates in the mass (or moment of inertia)weighted coordinates remain essentially unchanged on going from one single component to the mixed crystals, and to the other single component.

\section{ACKNOWLEDGMENT}

We thank the National Science Foundation for grant GP-8345, which enabled the departmental purchase of the Ramalog-Spex double spectrometer.

* Supported by NIH Grant NS08116.

$\dagger$ Present address: Department of Chemistry, State University of New York at Stony Brook, Stony Brook, N.Y. 11790.

1 I. M. Lifshitz, J. Phys. USSR 7, 215, 249 (1943) ; 8, 89 (1944).

${ }^{2}$ For a general review, see A. A. Maradudin, E. W. Montroll, G. H. Weiss, and I. P. Ipatova, Solid State Phys., Suppl. 3, 353 (1971), 2nd ed.

${ }^{3}$ For a general review, see N. F. Mott, Advan. Phys. 16, 49 (1967); N. F. Mott and W. D. Twose, ibid. 10, 107 (1961).

"See, for example, G. W. Robinson, Ann. Rev. Phys. Chem. 21, 429 (1970).

S. R. Ovshinsky, Phys. Rev. Letters 21, 1450 (1968).

' For example see Refs. 25, 16, 23, and 24.

${ }^{7}$ D. Adler, Critical Rev. Solid State Phys. 2, 317 (1971).

${ }^{8} \mathrm{H}$. K. Hong and R. Kopelman, I. Chem. Phys. 55, 724 (1971) ; Phys. Rev. Letters. 25, 1030 (1970); D. M. Hanson, J. Chem. Phys. 52, 3409 (1970).

'S. D. Colson, D. M. Hanson, R. Kopelman, and G. W. Robinson, J. Chem. Phys. 48, 2215 (1968).

${ }_{10}$ See Ref. 8.

11 W. Cochran and G. S. Pawley, Proc. Roy. Soc. A280, 1 (1964).

12 G. S. Pawley, Phys. Status Solidi 20, 347 (1967); G. S. Pawley and S. J. Cyvin, J. Chem. Phys. 52, 4073 (1970).

${ }_{13}$ G. Dolling and B. M. Powell, Proc. Roy. Soc. A319, 209 (1970).

14 (a) P. A. Reynolds, J. K. Kjems, and J. W. White, J. Chem. Phys. 56, 2928 (1972). (b) J. J. Rush, J. Chem. Phys. 47, 3936 (1967). Another recent paper on inelastic neutron scattering from benzene: K. W. Logan, S. F. Trevino, H. J. Prask, and J. D. Gault, J. Chem. Phys. 53, 3417 (1970).

${ }^{16} \mathrm{G}$. Dolling, Inelastic Scattering of Neutrons in Solids and Liquids, Proceedings of the Third Symposium (Bombay, India, 1964) (IAEA, Vienna, 1965), Vol. 1, p. 249.
${ }^{16}$ D. W. Taylor, Phys. Rev. 156, 1017 (1967).

17 For a definition of substitutional disorder, see, for example, Ref. 25 .

${ }^{18}$ C. Domb, A. A. Maradudin, E. W. Montroll, and G. H. Weiss, Phys. Rev. 115, 18, 24 (1959).

${ }^{10}$ See J. Hori, Spectral Properties of Disordered Chains and Lattices (Pergamon, Oxford, 1968), Chap. 3.

${ }^{20}$ P. Dean, Proc. Phys. Soc. (London) 73, 413 (1959); Proc. Roy. Soc. (London) A254, 507 (1960); A260, 263 (1961); P. Dean and J. L. Martin, ibid. A259, 409 (1960); P. Dean and M. D. Bacon, ibid. A283, 64 (1965); D. N. Payton III and W. M. Visscher, Phys. Rev. 154, 802 (1967) ; 156, 1032 (1967); 175, 1201 (1968); for application to exciton in disordered solids, see P. S. Juliene and S. I. Choi, J. Chem. Phys. 53, 2726 (1970), and H. K. Hong and R. Kopelman, J. Chem. Phys. 55, 5380 (1971).

21 M. Lax, Rev. Mod. Phys. 23, 287 (1951).

${ }^{22} \mathrm{~F}$. Yonezawa and T. Matsubara, Progr. Theoret. Phys. (Kyoto) 35, 357 (1966) ; 35, 759 (1966) ; 37, 1349 (1967); F. Yonezawa, ibid. 40, 734 (1968).

${ }^{23}$ P. Soven, Phys. Rev. 156, 809 (1967); 178, 1136 (1969).

24 Y. Onodera and Y. Toyozawa, J. Phys. Soc. Japan, 24, 341 (1968).

${ }^{25}$ B. Velicky, S. Kirkpatrick, and H. Ehrenreich, Phys. Rev. 175, 747 (1968).

${ }_{26}$ R. Kopelman, J. Chem. Phys. 47, 2631 (1967).

${ }^{27} \mathrm{H}$. K. Hong and G. W. Robinson, J. Chem. Phys. 52, 825 (1970) ; 54, 1369 (1971).

${ }^{28} \mathrm{O}$. A. Dubovskii and Yu. V. Konobeev, Fiz. Tverd. Tela, 12, 405 (1970) [Sov. Phys. Solid State 12, 321 (1970)].

${ }^{29} \mathrm{~J}$. Hoshen and J. Jortner, Chem. Phys. Letters, 5, 351 (1970) ; J. Chem. Phys. 56, 933 (1972); 56, 5550 (1972).

${ }^{30} \mathrm{H}$. K. Hong and R. Kopelman, "A Coherent Potential Theory for Interacting Bands: Phonons and Excitons in Substitutionally Disordered Molecular Crystals," J. Chem. Phys. (to be published).

${ }^{31}$ R. Kopelman, J. Chem. Phys. 44, 3547 (1966) ; E. R. Bernstein S. D. Colson, R. Kopelman, and G. W. Robinson, ibid. 48, 5596 (1968).

${ }_{32}$ P. H. Chereson, P. S. Friedman, and R. Kopelman, J. Chem. Phys. 56, 3716 (1972).

${ }^{33}$ See, for example, Ref. 36.

34 See Ref. 11.

${ }^{35}$ P. N. Prasad and R. Kopelman, J. Chem. Phys. 57, 863 (1972).

${ }^{36}$ I. Harada and T. Shimanouchi, J. Chem. Phys. 44, 2016 (1966).

${ }^{37} \mathrm{M}$. Ito and T. Shigeoka, Spectrochim. Acta 22, 1029 (1966).

${ }^{38}$ D. A. Oliver and S. H. Walmsley, Mol. Phys. 17, 617 (1969).

${ }^{39}$ E. R. Bernstein, J. Chem. Phys. 52, 4701 (1970).

${ }^{40}$ G. Taddei, H. Bonadeo, M. P. Marzocchi, and S. Califano, "Calculation of Crystal Vibrations of Benzene," J. Chem. Phys. (to be published).

41 E. R. Bernstein, S. D. Colson, R. Kopelman, and G. W. Robinson, J. Chem. Phys. 48, 5596 (1968); H. Winston and R. S. Halford, J. Chem. Phys. 17, 607 (1949); H. Winston, J. Chem. Phys. 19, 156 (1951).

${ }^{42} \mathrm{We}$ consider here a band to consist of four branches due to interchange-equivalent interactions. This classification is, of course, only approximate because bands are interacting.

${ }^{43}$ See Ref. 2, p. 50, Eq. (2.3.19). Our definition is slightly different from Eq. (2.3.19). This is done to be consistent with Eq. (5) in the text.

44 E. G. Cox, Rev. Mod. Phys. 30, 159 (1958); E. G. Cox, D. W. J. Cruickshank, and J. A. S. Smith, Proc. Roy. Soc. (London) A247, 1 (1958); G. E. Bacon, N. A. Curry, and S. A. Wilson, ibid. A279, 98 (1964).

${ }^{45}$ H. Bonadeo, M. P. Marzocchi, E. Castellucci, and S. Califano, J. Chem. Phys. 57, 4299 (1972).

${ }_{46}$ R. Kopelman, F. W. Ochs, and P. N. Prasad, J. Chem. Phys. 57, 5409 (1972). 